\title{
Searching for Planets Orbiting Distant Suns: Why Would You Look Through a Microscope?
}

\author{
Jacob L. Mey and Ben R. Oppenheimer \\ American Museum of Natural History, New York, NY 10024
}

A major effort in astrophysics today involves the search for and study of planets orbiting stars other than the Sun. This type of research demands large telescopes and complex optical systems that process the light of nearby stars. Such optical devices are some of the most precise imagers ever built and must differentiate starlight from that of the planets. The planets are between $10^{6}$ and $10^{12}$ times fainter than their parent stars. To differentiate objects with such incredible contrast requires exquisite optical surfaces and extremely precise control of the stellar light. At the American Museum of Natural History, the Lyot Project (http://lyot.org) involves such an imaging device. During development of the Lyot Project's "coronagraph," an instrument designed to block out as much starlight as possible without affecting the light from an orbiting planet, we investigated some of the microstructure in a set of the optics used. Our intention is to determine the effect of minute deformations in the optical surface on the final quality of the astronomical image. Typical astronomical imaging optics have surfaces that are precisely crafted with an rms deviation from the designed surface of less than $\lambda / 20$, where $\lambda$ is the wavelength of light $(\sim 1.6 \mu \mathrm{m}$ in the case of the Lyot Project). For exoplanet imaging, the light must be controlled at the level of $\lambda / 1000$ to $\lambda / 10,000$, in the propagation direction. In the spatial direction of a focal plane in the instrument, it is unclear what small pits or scratches contribute to degraded specular reflectivity and increased scattered light. To investigate this, we combine laser scanning confocal microscopy of optical surfaces with in-lab and real astronomical images.

In particular, we have investigated the surfaces around and the edges of small $(245-455 \mu \mathrm{m})$ holes in the "Focal Plane Mask" mirrors of the Lyot Project coronagraph with a Zeiss LSM-510 laser scanning confocal microscope (LSCM). We have developed and implemented a fast, non-destructive method of obtaining high resolution $(\sim 0.3 \mu \mathrm{m})$ images of surface features around the apertures. These optical apertures are located at the locus of the star in the astronomical image plane and are thus most likely to scatter light from the star. Knowledge of the spatial distributions and volumetric properties of these micro features is essential to understanding how they affect the final science image.

Our results are promising in that most of the submicron-sized features seen are resolved. The images can therefore be used for various spatial-analyses. We have produced 2D plane images for statistical distribution analysis and other general purposes. In addition, we have produced 3D images of the surface features and have obtained detailed knowledge of the depth or height into or above the mirror surface of these small pits. With high resolution three dimensional images of the surface features we intend to model the effects, perhaps allowing us to filter these on the final images obtained with the Lyot Project coronagraph. 


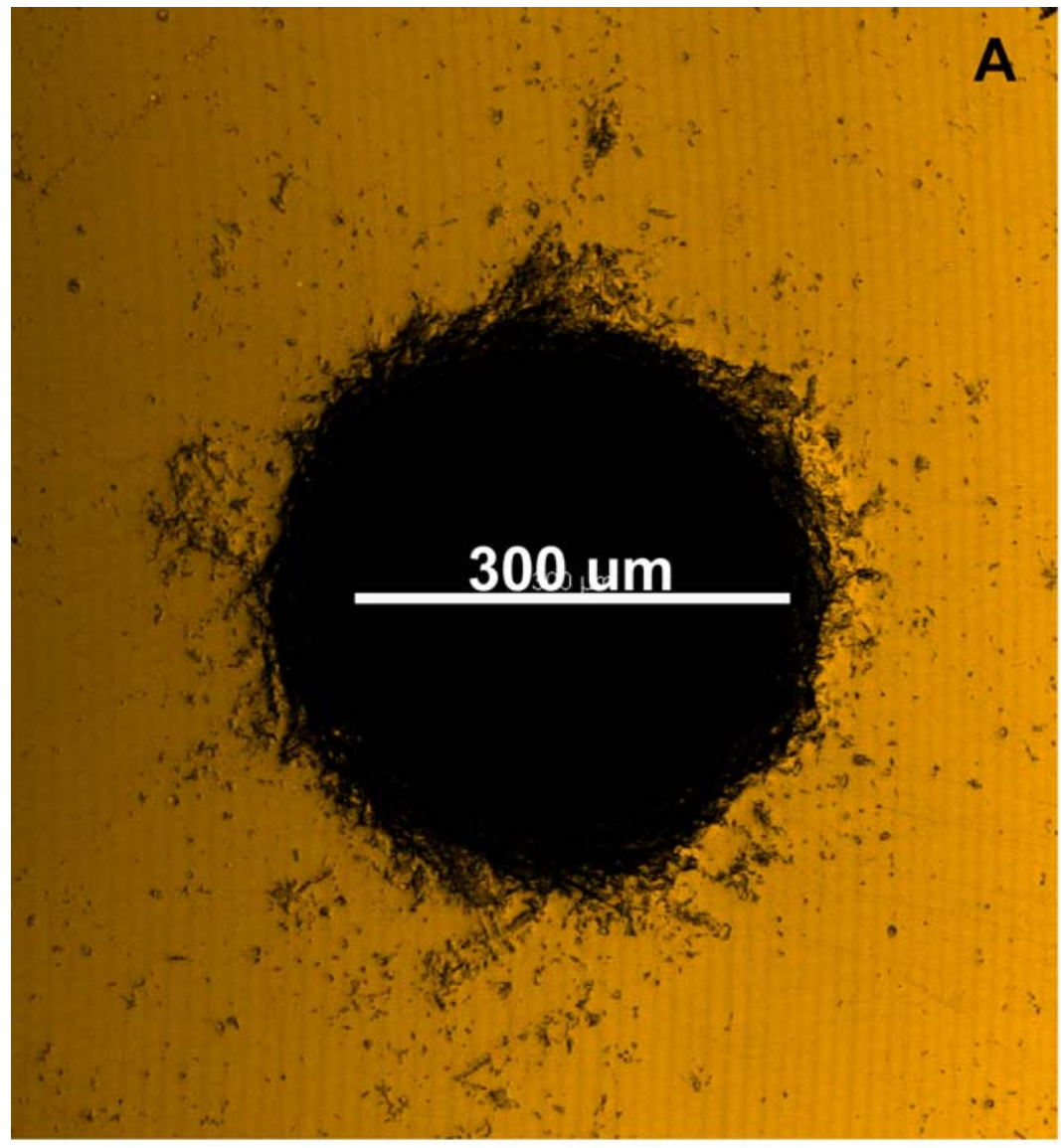

A. Confocal image of a gold mirror aperture (maximum intensity projection of a confocal stack). B. Optical image of gold mirror aperture.

C. Close up of B.

D. Relative reflection reduction plot of aperture edge in B. E. Size distribution of pits on mirror surface around aperture shown in B.

F. Data used in Figure E.

\begin{tabular}{|c|c|}
\hline Size bin um & coun \\
\hline 0.204 & 342 \\
\hline 0.399 & 55 \\
\hline 0.595 & 21 \\
\hline 0.790 & 33 \\
\hline 0.986 & 18 \\
\hline 1.182 & 17 \\
\hline 1.377 & 16 \\
\hline 1.573 & 14 \\
\hline 1.768 & 11 \\
\hline 1.964 & 8 \\
\hline 2.159 & 10 \\
\hline 2.355 & 0 \\
\hline 2.550 & 1 \\
\hline 2.746 & 3 \\
\hline 2.942 & 3 \\
\hline 3.137 & 9 \\
\hline 3.333 & 2 \\
\hline 3.528 & 3 \\
\hline 3.724 & 3 \\
\hline 3.919 & 1 \\
\hline 4.115 & 0 \\
\hline 4.311 & 0 \\
\hline 4.506 & 3 \\
\hline 4.702 & 0 \\
\hline 4.897 & 1 \\
\hline
\end{tabular}

